\title{
A Teoria Austríaca do Ciclo de Negócios na Perspectiva do Modelo GSMS
}

\author{
Antony P. Mueller*
}

\begin{abstract}
Resumo: O conteúdo analítico da teoria austríaca do ciclo de negócios permaneceu obscuro por falta de um modelo analítico compacto. O modelo GSMS (goods side - money side) é uma abordagem que deve tornar a teoria austríaca dos ciclos de negócios acessível para os que têm uma educação formal em economia, mas desconhecem os pormenores da teoria austríaca. O modelo GSMS difere da abordagem convencional da macroeconomia pelo não-intervencionismo e mostra que as políticas macroeconômica intervencionistas são as fontes de perturbações econômicas. Neste sentido, o modelo GSMS não é apenas uma ferramenta de política, mas a demonnstração de como a macroeconomia funciona.
\end{abstract}

Palavras-Chave: Escola Austríaca, Ciclos Econômicos, Macroeconomia, Modelo GSMS.

\section{The Austrian Business Cycle Theory in the Perspective of the GSMS Model}

\begin{abstract}
The Austrian business cycle theory remained unclear since it lacks a compact analytical model. The GSMS model (goods side - money side) is an approach that should make the Austrian theory of the business cycles accessible for those who have a formal education in economics, but are not sufficiently proficient with its details. The GSMS model differs from in its approach of macroeconomics by its non-interventionist orientation and it shows that macroeconomic interventionist policies are the sources of economic disturbances. In this sense, the GSMS model is not a policy tool, but a model that shows how the macroeconomy works.
\end{abstract}

Keywords: Austrian School of Economics, Business Cycles, Macroeconomics, GSMS Model.

Classificação JEL: B 53, E19, E32

\footnotetext{
*Antony P. Mueller é professor de Economia da Universidade Federal de Sergipe (UFS), presidente do The Continental Economics Institute, adjunct scholar do Ludwig von Mises Institute em Auburn, no Alabama, e membro do Conselho Editorial de MISES: Revista Interdisciplinar de Filosofia, Direito e Economia. Cursou a graduação, o mestrado, o doutorado e a livre-docência em economia na Universitat Erlangen-Nurnberg na Alemanha com estágios no exterior. Foi professor de Economia da Universitat Erlangen-Nurnberg FriedrichAlexander, professor associada da Radford University e Fulbright Scholar nos Estados Unidos, professor visitante da Universidad Francisco Marroquin (UFM) na Guatemala, professor da Universidade Federal de Santa Catarina (UFSC) e professor na Universidade de Caxias do Sul (UCS). É autor de artigos científicos publicados em diferentes periódicos acadêmicos, de capítulos de livros, de diversos artigos de opinião ou divulgação científica publicados em jornais ou em mídias eletrônicas, e das obras Sozialpolitik und Wirtschaftsordnung (Haat + Herchen, 1983), Portugal: Investitionsklima, Länderrisiko, Zahlungsfähigkeit (1986) e Die Kreditwürdigekeit souveräner Staaten. Methoden der Länderrisikoanalyse im inernationalen Kreditgeschäft der Banken (Universität Erlangen-Nürnberg, 1989). E-mail: antonymueller@gmail.com
} 
Não foram somente a crise financeira e as dificuldades da macroeconomia tradicional dos livros-texto que aumentaram o interesse pelas explicações consistentes e remédios da abordagem austríaca como um modelo alternativo. De fato, há décadas já cresce o interesse pela teoria austríaca dos ciclos econômicos. Na perspectiva da história do pensamento econômico, a teoria econômica austríaca faz parte integral da moderna teoria neoclássica. No entanto, embora tenha ocorrido uma ressurreição da economia neoclássica, a plena reabilitação da Escola Austríaca de Economia ainda está incompleta. Proeminentes economistas acadêmicos ainda se sentem confortáveis ao admitir ignorância sobre a abordagem austríaca e pronunciam, sem inibições, versões definitivas e errôneas da teoria austríaca do ciclo de negócios. Uma das razões pelas quais a teoria austríaca dos ciclos econômicos ainda não faz parte do mainstream decorre do fato de não ter um modelo analítico convincente. Há necessidade de um modelo que apresente as principais características da teoria austríaca da teoria do ciclo de negócios de maneira que facilite a compreensão, particularmente para aqueles economistas acadêmicos que não conhecem muito bem a economia austríaca, mas estão familiarizados com a teoria macroeconômica convencional, tal como está representada nos livros-texto populares.

Um modelo não pode contar toda a história; e este também é o caso do modelo GSMS. A principal função desse modelo macroeconômico é mostrar as ligações entre os principais setores da economia. Como tal, o modelo GSMS serve como um guia para o ensino e a pesquisa e oferece um quadro para a discussão crítica de conceitos de política econômica. Para dar vida ao modelo, precisamos da aplicação da praxeologia ${ }^{1}$.

${ }^{1}$ IORIO, Ubiratan. Ação, Tempo e Conhecimento: A Escola Austríaca de Economia. São Paulo: Instituto Ludwig von Mises Brasil, 2011.

\section{I - Estrutura Básica do Modelo do Ciclo de Negócios}

No ponto de partida da teoria austríaca dos ciclos econômicos está a questão de como uma multiplicidade de agentes econômicos poderia cometer simultaneamente o mesmo tipo de erro. Como é possível que os empresários desviem investimentos em larga escala? Por que os consumidores superestimam sistematicamente os seus futuros fluxos de rendimentos? Rejeitando explicações psicológicas, os economistas austríacos argumentam que os agentes econômicos devem ter seguido, via de regra, orientações que, enquanto provarem ser confiáveis, no início do ciclo, transmitem sinais errados ${ }^{2}$. A taxa de juros, em particular, é falsificada. As forças de mercado dificilmente podem ser responsáveis por extremas oscilações de preços, de modo específico, na formação da taxa de juros, que orienta a alocação intertemporal. Assim, a teoria destaca o papel das autoridades monetárias. A teoria austríaca dos ciclos econômicos afirma que com mais liquidez na economia do que a compatível com a poupança autêntica, as intenções dos consumidores se chocam com as dos investidores. Quando as autoridades monetárias fixam a taxa de juros de mercado abaixo da taxa natural, a demanda por fundos emprestáveis aumenta e passa a exceder a poupança autêntica. Esta discrepância atrapalha a percepção da disponibilidade de recursos e instiga uma má alocação de investimentos. Com a taxa monetária de juros abaixo do nível de livre mercado, os planos de investimento entram em colisão com os planos de consumo. Enquanto os consumidores não tiverem a intenção de reduzir o consumo, os empresários desejarão investir mais. A economia fica estimulada, mas o que acontece

\footnotetext{
2 HAYEK, F. A. Price Expectations, Monetary Disturbances and Malinvestments (1933). In: Profits, Interest and Investment and Other Essays on the Theory of Industrial Fluctuations. Clifton: August M. Kelly Publishers, 1975. (Reprints of Economic Classics). p. 13556, esp. p. 142.
} 
é um boom artificial ${ }^{3}$. À medida que a atividade econômica se move em direção à utilização da capacidade total, subirão os custos. Quando as taxas de juros e salários se adaptam ao grau mais elevado de escassez dos fatores de produção e quando os agentes econômicos, consequentemente, revisam as expectativas, as taxas de lucro ficam sob pressão e indicam que os cálculos de rentabilidade foram o resultado de uma superestimação da capacidade da economia para crescer.

Balanços deterioram tanto para os devedores como para os credores. É apenas no boost que os agentes econômicos percebem que têm menos riqueza do que esperavam. Sentimentos mudam do otimismo ao pessimismo, mas não é a psicologia que impulsiona o ciclo de negócios. Mudanças de humor acompanham os altos e baixos do ciclo. $\mathrm{O}$ motor real do ciclo de negócios é a expansão e a contração da liquidez macroeconômica: a inflação e a deflação em seu significado original, como expansões e contrações da massa monetária.

No início do ciclo, a expansão monetária nos mercados financeiros acelera a expansão econômica com uma bonança de crédito. Este boom artificial acontece por meio de uma acumulação de maus investimentos. Ambos, a expansão e a contração desenvolvem as próprias dinâmicas como processos de auto -alimentação cumulativa. A criação monetária está no início do boom, enquanto contração monetária marca o bust ${ }^{4}$. Consequentemente, o foco do presente modelo encontra-se nos efeitos das variações dos meios de pagamento sobre o produto e os preços.

\section{II - EsBoço Do Modelo GSMS}

O que move a economia não são agregados e médias, mas a ação humana indi-

\footnotetext{
${ }^{3}$ GARRISON, Roger. Time and Money: The Macroeconomics of Capital Structure. London: Routledge, 2000. (Routledge Foundations of the Market Economy).

${ }^{4}$ HUERTA DE SOTO, Jesus. Money, Bank Credit, and Economic Cycles. Auburn: The Ludwig von Mises Institute, $3^{\text {rd ed., } 2012 .}$
}

vidual $^{5}$. Contar a história em termos de ação humana está no domínio principal da economia austríaca. A história completa requer ser contada em termos de ação humana. No entanto, é o modelo que oferece o arcabouço do enredo. $\mathrm{O}$ critério para um bom modelo é como esse pode servir para estruturar a análise. Um modelo deixa muito espaço para o usuário preencher sua estrutura com a vida em termos de ação humana.

A teoria quantitativa da moeda é a base para a presente abordagem. Esta teoria remonta a Nicolau Copérnico (1473-1543) no século XVI e a David Hume (1711-1776) no século XVIII. Ao longo da sua história, a teoria quantitativa da moeda tem experimentado um ciclo próprio de altos e baixos.

A teoria quantitativa relaciona a massa monetária $(M)$ com as transações $(T)$ e a renda nacional $(Y)$, e vincula dinheiro $(M)$ com o conceito de velocidade de circulação $(V)$ ou o saldo de caixa $(k)$.

A distinção na versão transação Chicago/ Fisher é

$$
\begin{gathered}
M x V_{T}=P x T \\
M x V_{Y}=P x Q=Y
\end{gathered}
$$

e na versão de Cambridge

$$
M=k P Q=k Y
$$

Anthony J. Evans e Robert Thorpe identificam como a versão austríaca ${ }^{6}$ a equação do tipo

$$
M=k P T
$$

Para a abordagem que aqui se apresenta, no entanto, o modelo faz a distinção fundamental entre o "lado de bens" (GS) e o "lado da moeda" (MS) da economia. Como tal, a equação básica para o modelo GSMS é

$$
\frac{M V}{P}=\mathrm{Q}
$$

${ }^{5}$ MISES, Ludwig von. Human Action. A Treatise on Economics. Auburn: The Ludwig von Mises Institute, 2010.

6 EVANS, Anthony J. \& THORPE, Robert. The (Quantity) Theory of Money and Credit. Review of Austrian Economics, Vol. 26, No. 4 (2013): 463-81. 
O modelo GSMS distingue entre o "lado de bens" (GS) e o "lado da moeda" (MS) da economia, com base em uma reformulação da equação de troca para separar as variáveis monetárias e as variáveis para a produção real. Assim aparece o "lado ao dinheiro" como a liquidez macroeconômica real $\left(\frac{M V}{P}\right)$ e o "lado de bens" como produção (Q).

Com uma oferta de dinheiro fixa e uma velocidade constante, a relação entre os preços $(\mathrm{P})$ e produto $(Q)$ forma uma hipérbole. Em sua representação gráfica (curva ML na Figura 1), o estoque de dinheiro em circulação representa a liquidez macroeconômica $(M L)$ e é composto de dinheiro como meio de pagamento $(M)$ multiplicado pela sua velocidade de circulação $(V)$.

Tendo em conta que a renda nacional nominal $(Y)$ é igual à produção real $(Q)$ multiplicada pelo nível de preços $(P)$, a renda nominal é o retângulo da área com o nível de preços e de produção como os seus lados. A fim de captar a renda nacional nominal, o modelo básico experimenta uma extensão em forma de

$$
M x V=Q x P=Y
$$

A extensão da equação pelos componentes de despesas para o consumo $(C)$, investimento $(I)$, gastos do governo $(G)$ exportações $(E X)$ e importações (IM) revela como a análise keynesiana padrão relaciona-se com o lado da moeda e o lado de bens da economia.

$Q \times P=Y=C+I+G+N X=P_{c} \times Q_{c}+P_{I} \times Q_{I}+P_{G} \times Q_{G}+P_{E X} \times Q_{E X}-P_{I M} \times Q_{I M}$

Da mesma forma pode-se estender do lado esquerdo, a fim de incluir as fontes de liquidez. Liquidez macroeconômica $(M L)$ no lado monetário da equação é o resultado da base monetária $(M B)$ multiplicado pelo multiplicador bancário $\left(m_{b}\right)$ e da velocidade de circulação $(V)$.

$$
M L=M B \times m_{b} \times V
$$

Nesta fase, o modelo inclui a conta de moeda, que começa com a base monetária os preços e os bens produzidos e continua com a estrutura de produção. Limitando o modelo para uma economia fechada a fórmula é $\mathrm{BM} \times \mathrm{m}_{\mathrm{b}} \times \mathrm{V}=\mathrm{Q} \times \mathrm{P}=\mathrm{Y}=\mathrm{C}+\mathrm{I}+\mathrm{G}=\mathrm{P}_{\mathrm{C}} \times \mathrm{Q}_{\mathrm{C}}+\mathrm{P}_{\mathrm{I}} \times \mathrm{Q}_{\mathrm{I}}+\mathrm{P}_{\mathrm{G}} \times \mathrm{Q}_{\mathrm{G}}$
Em termos de atores e decisões, a equação contém, da esquerda para a direita, o banco central, que decide sobre a base monetária; os atores do mercado financeiro, que determinam o multiplicador bancário; e todos os agentes econômicos que decidem sobre investimentos em dinheiro. No lado direito da equação, a caixa-preta da produção total $(Q)$, o nível de preços $(P)$ e a renda nacional nominal $(Y)$ abre-se em termos de preços relativos, tais como PC/PI ou PI/ PQ, e assim por diante, em nível da agregação intermediário. Em forma detalhada, a extensão do modelo para além da agregação intermediária em termos de consumo, investimento e governo.

Enquanto a fronteira de produção natural depende da eficiência dos fatores de produção, a produção atual vem de empresas como entidades onde os empresários e gestores combinam trabalho $(L)$, capital $(K)$ e know-how $(A)$, a fim de ter bens $(q)$ para vender a um preço $(p)$ com a intenção de obter lucro $(\Pi)$.

Definindo custos em termos da taxa de salário $(w)$ e da quantidade de trabalho $(L)$, juntamente com a taxa de juros (i) e o estoque de capital $(K)$, segue que, ceteris paribus, tanto um preço mais elevado $(p)$ ou maior quantidade vendida $(q)$ vão aumentar o lucro $(\Pi)$. Da mesma forma o lucro aumenta com uma menor taxa de salário $(w)$ e de juros (i). Maior produtividade $(A)$ mostra-se como uma redução de

\section{Figura 1 - Modelo básico GSMS}

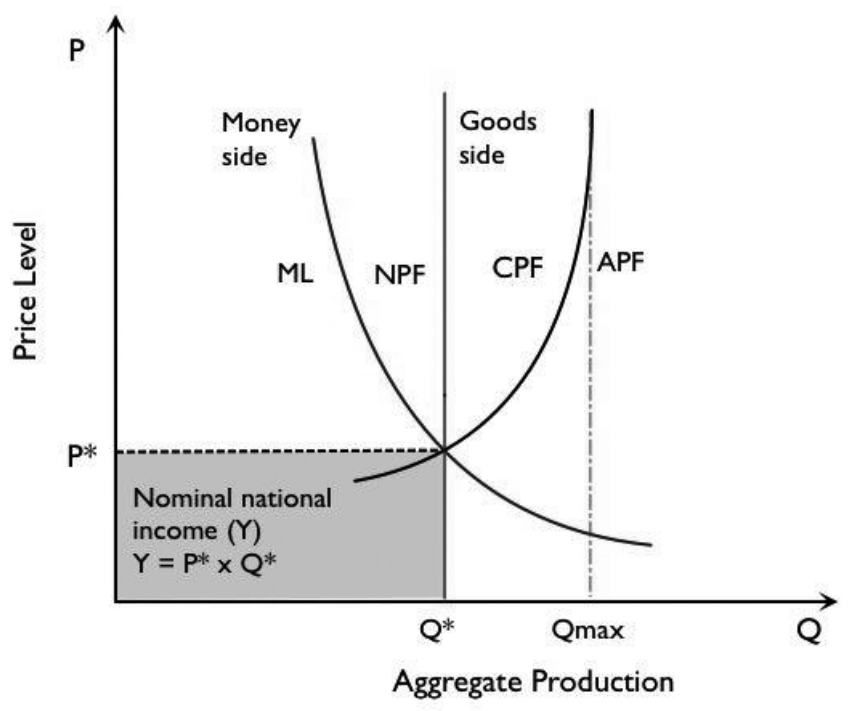


custos quando o mesmo tamanho do output pode ser obtido com menor insumo ou um maior output pode ser obtido com o mesmo insumo. No nível empresarial, o motivo de procurar progresso tecnológico resulta do efeito da eficiência sobre o lucro.

$$
\Pi=(p \times q)-(w L+i K-\alpha A)
$$

Omodelo GSMS distingue entre uma fronteira da produção "natural" (NPF) e a fronteira de produção "cíclica" (CPF). A distinção entre o curso normal de negócios - representado pela curva NPF - e da atividade de negócios excepcional ou acima ou abaixo deste nível - representado pela curva CPF - é fundamental para a condução de uma empresa. Mais se aproxima a atividade econômica do limite da capacidade, mais os custos monetários e de oportunidade vão subir como resultado da crescente escassez, e mais será necessário obter preços mais elevados como compensação para manter a rentabilidade. Da mesma forma, quando a atividade cai abaixo de seu nível normal, existe capacidade não utilizada e a concorrência derruba os preços. Diferente da fronteira de produção cíclica $(C P F)$, que indica a variação da produção atual em relação ao nível de preços, a fronteira de produção natural (NPF) é independente do nível de preços e muda de acordo com as mudanças de quantidade e qualidade dos fatores de produção.

\section{III - DinÂMicA do MoDElo GSMS}

O modelo GSMS é composto de um lado monetário (MS) e de um lado de bens (GS) com a diferenciação entre a fronteira de produção natural (NPF), a fronteira de produção absoluta $(A P F)$ e a fronteira de produção cíclica $(C P F)$.

Em forma dinâmica, com $g$ designando a taxa de variação percentual, a equação de troca se lê como

$$
\mathrm{g}_{\mathrm{M}}+\mathrm{g}_{\mathrm{V}}=\mathrm{g}_{\mathrm{Q}}+\pi
$$

Dado que a liquidez macro $(M L)$ é composta de dinheiro multiplicado pela sua velocidade, a equação torna-se

$$
\pi=g_{M L}-g_{Q}
$$

Nesta forma reduzida, alterações de preços resultam da relação entre o crescimento da liquidez e crescimento econômico real ( $g M L-$ $g Q)$, enquanto que ao aplicar os determinantes elaborados anteriormente, a equação para a inflação de preços torna-se:

$$
\pi=\left(g_{M B}+g_{m h}+g_{v}\right)-\left(g_{Q n}+g_{Q c}\right)
$$

A fim de obter a estabilidade de preços com uma taxa de inflação de zero $(\pi=0)$, a condição assim é:

$$
\left(g_{M B}+g_{m b}+g_{v}\right)=\left(g_{Q n}+g_{Q c}\right)
$$

A taxa de desemprego é inversa à expansão da economia, ou seja, o crescimento cíclico, enquanto o crescimento econômico natural (deslocamento da curva NPF para a direita) vem com um emprego estável ou om uma taxa de emprego que se mantém em seu nível natural. Portanto, a taxa de desemprego atual é uma função da atividade econômica, enquanto que a taxa natural de desemprego coincide com a fronteira de produção natural $(N P F)$. Como a renda nacional nominal $(Y)$ é o produto da produção real e do nível de preços como especificado pelo modelo, a taxa de crescimento da renda nominal $(g Y)$ é dado por

$$
\mathrm{g}_{\mathrm{Y}}=\mathrm{g}_{\mathrm{Q}}+\pi=\mathrm{g}_{\mathrm{Qn}}+\mathrm{g}_{\mathrm{QC}}+\pi
$$

Essas equações fornecem as ferramentas para compor uma tabela de constelações macroeconômicas composta pelas variáveis que aparecem no conjunto das equações básicas do modelo GSMS. Essas constelações macroeconômicas na Tabela 1 como mudanças da fronteira natural e da fronteira cíclica da produção, juntamente com a curva de liquidez macroeconômica, têm como base mudanças potenciais e reais das variáveis conforme a equação dinâmica estendida de troca.

$$
\pi=\left(g_{M B}+g_{m b}+g_{v}\right)-\left(g_{Q n}+g_{Q c}\right)
$$


TABela 1 - O MOdelo GSMS COMO UMA FERRAMENTA DE CLASSIFICAÇÃO DAS CONSTELAÇÕES MACROECONÔMICAS

\begin{tabular}{|l|c|c|c|}
\hline & $\begin{array}{c}\text { Liquidez } \\
\text { Macroeconômica } \\
(\mathrm{ML})\end{array}$ & $\begin{array}{c}\text { Fronteira } \\
\text { Ciclica da } \\
\text { Produção (CPF) }\end{array}$ & $\begin{array}{c}\text { Fronteira } \\
\text { Natural da } \\
\text { Produção } \\
\text { (NPF) }\end{array}$ \\
\hline PLG & 0 & $\searrow$ & $\rightarrow$ \\
\hline MPI & $\nearrow$ & $\uparrow$ & 0 \\
\hline MHI & $\nearrow$ & $\downarrow$ & $\leftarrow$ \\
\hline MPD & $\swarrow$ & $\downarrow$ & 0 \\
\hline DD & $\swarrow$ & $\uparrow$ & $\leftarrow$ \\
\hline IS & 0 & $\uparrow$ & 0 \\
\hline IB & $\nearrow$ & $\downarrow$ & 0 \\
\hline
\end{tabular}

PLG: Productivity-led (deflationary) economic growth - crescimento econômico deflacionário baseado em avanços da produtividade

MPI: Monetary price inflation - inflação monetária de preços

MHI: Monetary hyperinflation - hiperinflação monetária

MPD: Monetary price deflation - deflação monetária de preços

DD: Deflationary depression - depressão deflacionária IS: Inflationary stagnation (stagflation) - estagnação inflacionária (estagflação)

IB Inflationary boom - boom inflacionário

As setas indicam a direção dos movimentos das curvas

O modelo GSMS serve para identificar configurações macroeconômicas específicas e orientar a análise. A seguir a Tabela 2 mostra as variáveis do modelo, a fim de analisar as ligações entre as diferentes partes da macroeconomia.

\section{Tabela 2 - Constelações macroeconômicas EM TERMOS DAS VARIÁVEIS DO MODELO GS / MS}

\begin{tabular}{|l|c|c|c|c|c|c|c|c|}
\hline & $\mathrm{g}_{\mathrm{MB}}$ & $\mathrm{g}_{\mathrm{mb}}$ & $\mathrm{g}_{\mathrm{v}}$ & $\mathrm{g}_{\mathrm{Qc}}$ & $\mathrm{g}_{\mathrm{On}}$ & $\Pi$ & $\mathrm{Q}$ & $\mathrm{Y}$ \\
\hline PLG & 0 & 0 & 0 & + & + & - & + & 0 \\
\hline MPI & + & + & 0 & 0 & 0 & + & 0 & + \\
\hline MHI & + & + & + & - & - & + & - & + \\
\hline MPD & - & - & - & - & 0 & - & - & - \\
\hline DD & - & - & - & - & - & - & - & - \\
\hline IS & 0 & 0 & 0 & - & - & + & - & - \\
\hline IB & + & + & + & + & 0 & + & + & + \\
\hline
\end{tabular}

As tabelas 1 e 2 fornecem uma amostra de configurações macroeconômicas típicas7. O modelo é capaz de capturar também constelações macroeconômicas específicas, como a atual Grande Recessão, por exemplo, que aparece como um forte crescimento da base monetária, o que não se transforma em equivalente maior liquidez por causa de um baixo multiplicador bancário e uma taxa de crescimento da velocidade negativa. Consequentemente, o efeito da política monetária sobre a produção e os preços é impotente.

\section{IV - Análise do Ciclo de Negócios}

Em termos do modelo GSMS, o crescimento econômico deflacionário (Figura 2a) representa o equilíbrio dinâmico do sistema. A produtividade se desenvolve de forma lenta e permite a adaptação contínua das expectativas. Em contraste a esta "deflação bondosa", uma "deflação maliciosa" representa uma queda em uma depressão deflacionária como consequência de um boom inflacionário anterior que geralmente ocorre como um colapso comprimido em um curto espaço de tempo (Figura 2d). O colapso inesperado de liquidez rompe contratos econômicos em termos nominais, e não deixa tempo suficiente para a revisão.

Os quatro gráficos apresentam uma análise sequencial do ciclo de negócios no contexto do modelo GSMS. Ceteris paribus, o aumento da produtividade levaria ao crescimento econômico deflacionário (Figura 2a). Tal expansão viria com o aumento do poder de compra do dinheiro. As autoridades monetárias provocam um boom inflacionário quando tentam manter a "estabilidade de preços" e assim expandem a oferta de dinheiro que movimenta a atividade econômica além da fronteira de produção natural (Figura 2b).

\footnotetext{
7 MUELLER, Antony P. Beyond Keynes and the Classics. Outline of the Goods Side/Money Side Model of the Business Cycle and Macroeconomic Configurations. Social Science Research Network SSRN, February 2014. http://papers.ssrn.com/sol3/cf_dev/AbsByAuth.cfm?per_id=2181390
} 
Figura 2A - Expansão Econômica da Produtividade

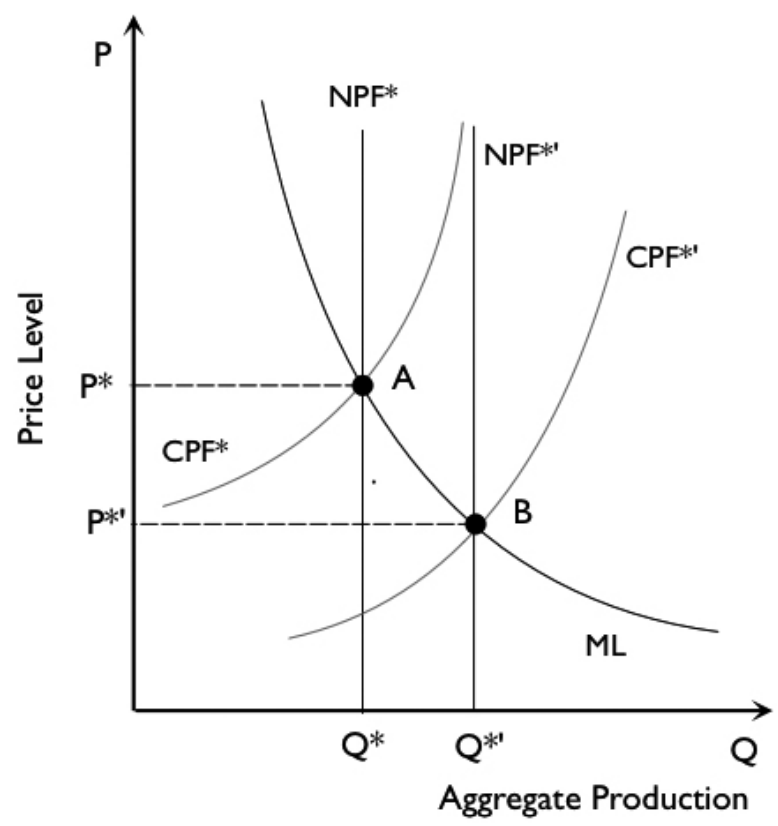

Figure 2C - Contração Inflacionária

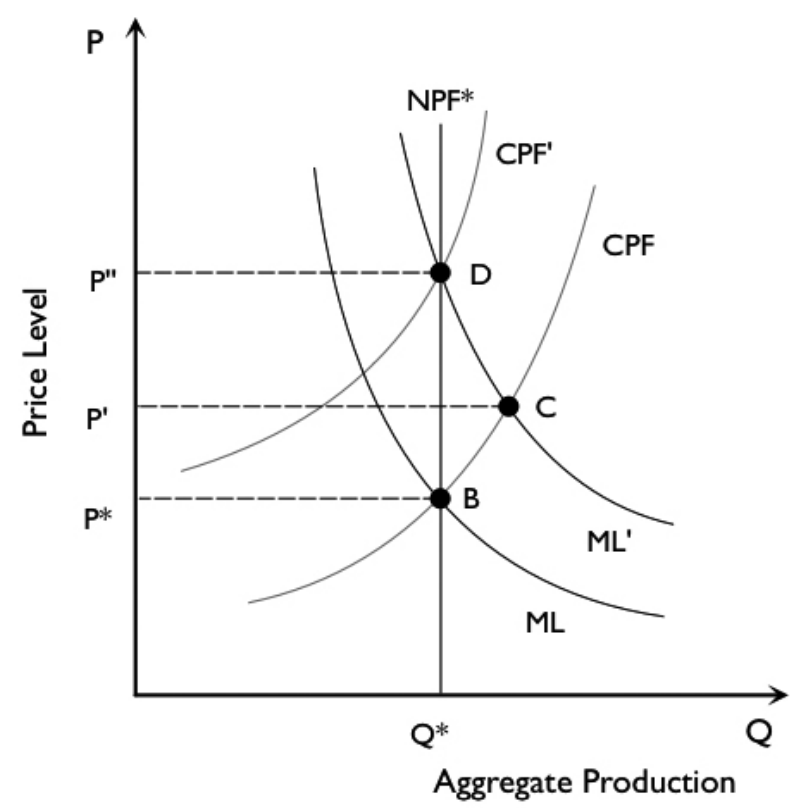

A atividade econômica que excede o nível natural $\left(\mathrm{Q}>\mathrm{Q}^{*}\right)$ vai aumentar os preços de produção como consequência de maior escassez, dado o aumento da quantidade de liquidez macroeconômica que está disponível. No devido tempo, a fronteira de produção cíclica, que de outra forma teria caído, se move para a esquerda em direção à sua posição original $\left(\mathrm{CPF}^{*}\right)$. Nesta fase, a inflação mone-
FIGURA 2B - BoOM INFLACIONÁRIO

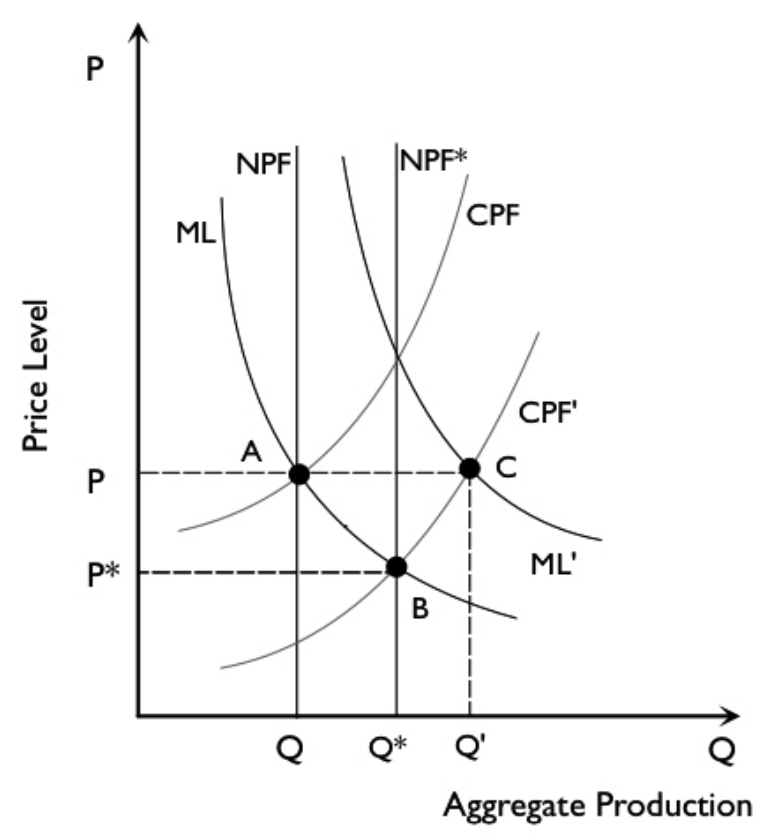

Figura 2D - Contração Deflacionária

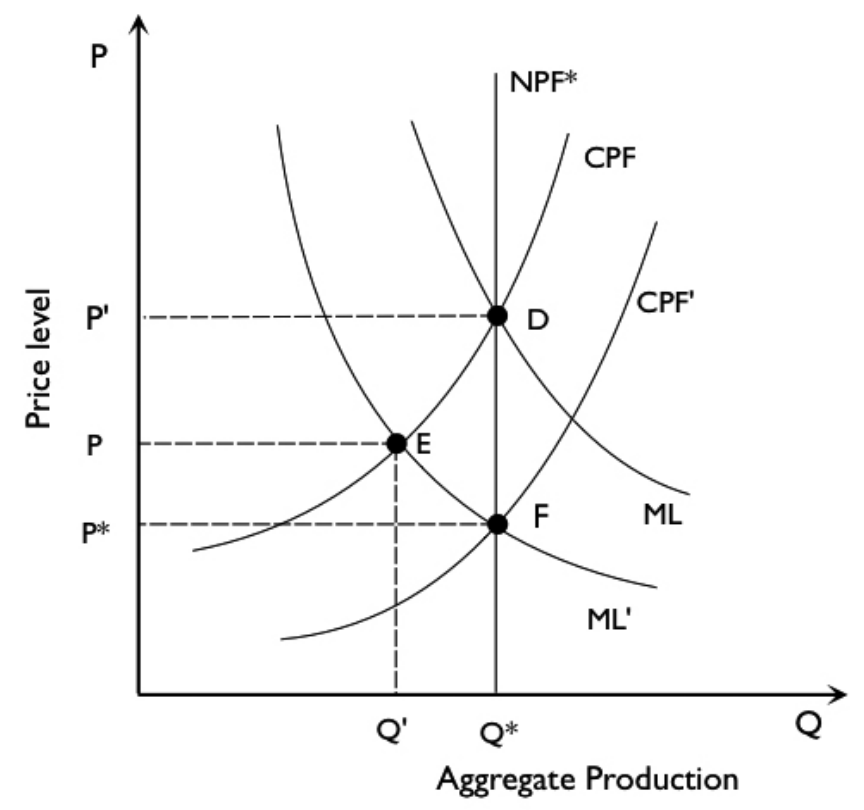

tária escondida torna se numa inflação de preços aberta quando a economia se move em direção à estagflação (Figura 2c).

O boom inflacionário que se transformou em um bust acarreta dívidas incobráveis. Quando os bancos centrais tentam inflacionar de novo na sua luta contra a contração da liquidez deflacionária, estão cometendo os mesmos erros duas vezes. A primeira vez, 
quando eles impedem o efeito da deflação bondosa do aumento da produtividade e seu efeito da uma deflação bondosa instigando o boom inflacionário (passagem de A para C na Figura 2b). A segunda vez, quando o bust chega, quando os formuladores de políticas monetárias enfrentam deflação maliciosa e impedem agora a sua rápida eliminação, como consequência do esforço dos bancos centrais para voltar a inflar a economia (Figura 2d).

As políticas macroeconômicas que fabricam expansão monetária e empurram a taxa de juros abaixo da taxa de mercado livre estimulam a expansão econômica além da fronteira de produção natural da economia. $\mathrm{O}$ erro original é o boom inflacionário e não as contrações deflacionárias. Sem intervenção, o bust serve para corrigir os erros cometidos no boom inflacionário. A contração deflacionária é a consequência do boom inflacionário. Em vez de acelerar a recuperação, o estímulo monetário e fiscal vai dificultar a retomada do crescimento econômico autossustentado e realmente prolongar a estagnação. Em termos do modelo (Figura 2d), o caminho natural da adaptação iria de $\mathrm{D}$ para $\mathrm{E}$ e daí para $\mathrm{F}$. Tentando evitar este processo da adaptação, as autoridades monetárias cometem no bust o mesmo erro que se iniciou o boom. Assim, a economia se mantém na estagnação ou depressão no ponto E da Figura 2d, abaixo do nível do produto natural $\left(\mathrm{Q}^{\prime}<\mathrm{Q}^{*}\right)$.

Desde a revolução keynesiana, a macroeconomia encontrou sua principal razão em fornecer ferramentas para os tomadores das decisões políticas. O foco central da macroeconômica moderna é a tese de que, quando deixada por conta própria, a economia capitalista vai mergulhar em depressão por causa de grandes oscilações do ciclo de negócios. A política macroeconômica deve estabilizar a economia e evitar que ela caia em depressão deflacionária. À luz do modelo GSMS, no entanto, os conceitos de política macroeconômica intervencionistas são falhos. Em vez de estabilizar a economia, as intervenções de política macroeconômica tendem a ser ineficazes ou mesmo contribuir para a instabilidade.

\section{V - O MODELO GSMS E O PRINCÍPIO}

\section{DA DEMANDA EFETIVA}

O princípio da demanda efetiva, que está no cerne da abordagem keynesiana básica, diz que a demanda agregada determina a produção de agregados. A magia da economia keynesiana existe na fórmula que a identificação da causa promete a cura. Quando a falta de demanda efetiva é a causa da depressão, tudo o que é preciso para sair do bust é produzir a expansão econômica com a ajuda de uma demanda adicional. Se as empresas privadas não podem exercer demanda suficiente, o governo deve fazê-lo. Dívida pública como resultado do "deficit spending" aparece como a chave para a prosperidade. Quando John Hicks (1904-1989), que na verdade era muito simpático às ideias austríacas, apresentou a alegação keynesiana em um modelo gráfico8, de fato, fez isso mais como um capricho do que por convicção9, pois não apresentou nenhum argumento para o triunfo do keynesianismo. Nesse espírito, a abordagem ISLM-AS atualmente dominante na teoria econômica tradicional é mais uma ferramenta de engenharia do que um modelo da economia ${ }^{10}$.

Apesar de triunfo, o princípio básico da ideia keynesiana falhou. A síntese do modelo ISLM, mesmo junto com a oferta agregada, como no modelo ISLM-AS não elimina as falhas, mas acrescenta novas incompatibilidades11. O modelo GSMS pode ajudar a revelar

${ }^{8}$ HICKS, John. Mr. Keynes and the 'Classics'. A Suggested Interpretation. Econometrica, Vol. 5, No. 2, (April 1937): 147-59.

9 Idem. IS-LM: An Explanation. Journal of PostKeynesian Economics, Vol. III, No. 2 (1980/1981): 13954.

${ }^{10}$ MANKIW, Gregory N. The Macroeconomist as Scientist and Engineer. Journal of Economic Perspectives, Vol. 20, No. 4 (Fall 2006): 29-46.

${ }^{11}$ COLANDER, David. The Stories We Tell: A Reconsideration of AS/AD Analysis. Journal of Economic Perspectives, Vol. 9, No. 3 (Summer 1995): 169-88. 
as contradições e omissões da abordagem keynesiana. Há uma confusão profunda em modelos keynesianos padrão, como o regime ISLM sobre quais variáveis representam os valores "reais" e quais os valores "nominais". Modelos keynesianos sofrem de ofuscação em relação ao tempo em que as variações da demanda agregada determinam a produção. No entanto, embora com mais dinheiro na economia, a demanda de fato pode aumentar imediatamente, enquanto a produção real de bens e serviços leva tempo para sua realização e está confrontada com uma infinidade de situações de escassez específica. Além disso, também a oferta agregada do modelo de oferta e demanda agregada (AS/AD), que viria a substituir e complementar o modelo ISLM, não poderia acabar com as inconsistências fundamentais. Enquanto o modelo ISLM só funciona como pretendido quando os preços são constantes, as variações do nível de preços determinam o lado da demanda agregada do modelo AS/AD.

Nas primeiras décadas da revolução keynesiana houve uma negação quase completa da moeda como um fator macroeconômico até o início da contrarrevolução monetarista. Contudo, o que a maioria dos keynesianos não quis ver era que o dinheiro de fato também é importante, juntamente com a política fiscal. Gastar é um conceito monetário e o termo "despesa" inclui o lado real e o lado nominal ao mesmo tempo por incluir preços. Os déficits orçamentários implicam a criação de mais dinheiro que o governo gasta. Desta forma, o princípio da demanda efetiva mostra-se no modelo de GSMS como um deslocamento da curva ML para cima, para a direita. No entanto, diferente da "cruz keynesiana" ou do modelo ISLM, a análise GSMS atrai automaticamente a atenção para a questão sobre qual grau de estímulo fiscal afetaria o nível de preços em distinção à produção real.

Em contraste com a abordagem keynesiana, o modelo GSMS faz, em primeiro lugar, uma clara distinção entre o lado do dinheiro e o lado de bens da economia. Em segundo lugar, com a sua distinção crucial entre a fronteira de produção cíclica e natural, o modelo GSMS descarta a ilusão dos modelos keynesianos de que o crescimento econômico sustentável poderia simplesmente vir de mais gastos. Em terceiro lugar, o modelo GSMS abre as caixas-pretas de ambos, tanto do keynesianismo quanto do monetarismo. Enquanto o lado da produção evapora completamente no monetarismo, o keynesianismo fabrica a ilusão identificar gastos do governo com produção.

\section{Conclusão}

A análise GSMS diferencia sistematicamente entre as despesas que vão para os preços e aquela parte que entra na produção real. Quanto à política macroeconômica, o modelo GSMS é não-intervencionista. Ao deixar a deflação benéfica acontecer, a deflação maliciosa não vai aparecer. $\mathrm{O}$ modelo GSMS destaca a quintessência da teoria austríaca dos ciclos econômicos, segundo a qual as expansões econômicas inflacionárias são o resultado de estímulos monetários (que incluem déficit público) que provocam booms insustentáveis que revertam em busts. Embora as medidas políticas expansionistas funcionem para iniciar um boom, elas são ineficazes no bust porque a depressão deflacionária é a consequência direta da expansão inflacionária no início do ciclo e depois porque a economia sofre de um excesso de dívidas incobráveis como resultado de investimentos mal direcionados. Quanto a suas implicações políticas, o modelo GSMS, como escrevemos linhas atrás, é não intervencionista. Portanto, o modelo GSMS está imune à crítica de Lucas, segundo a qual as correlações entre as variáveis agregadas observadas nos dados macroeconômicos tenderiam a mudar sempre que a política macroeconômica mudasse, porque a própria ineficácia das políticas macroeconômicas está no cerne desse modelo. cos 
